\title{
À propos des trajectoires et des défis contrastés des régions québécoises
}

\author{
Mario Polèse * \\ Laboratoire d'analyse spatiale et d'économie régionale (LASER) \\ INRS Urbanisation, Culture et Société
}

\section{Introduction}

Cet article est d'abord une synthèse d'une communication présentée au Colloque de l'Association de Science Régionale de Langue Française (ASRDLF) à l'Université du Québec à Rimouski en août 2008 dans la session spéciale «École québécoise de sciences régionales ». La communication a fait l'objet d'une publication ${ }^{1}$ que j'invite le lecteur à consulter (Polèse 2009) pour les analyses et les données sur l'évolution territoriale de l'emploi, sur lesquelles reposent souvent mes affirmations. D'autres affirmations reposent

\section{La remétropolisation de Montréal}

Le premier indice de la maturité de l'économie québécoise est la tertiarisation avancée de sa plus grande ville. Toutes proportions gardées, l'activité manufacturière est aujourd'hui davantage présente à l'extérieur de la région montréalaise. En contrepartie, la région montréalaise s'affirme de plus en plus par la concentration des emplois dans le tertiaire supérieur: services informatiques, services comptables, autres services professionnels, radio-télédiffusion, multimédia, commercialisation, gestion, maisons d'édition, finance, etc.

Cette affirmation de Montréal comme métropole moderne est l'aboutissement d'une deuxième transformation, raison pour laquelle nous parlons de « re » métropolisation. Dans les années 1970, Montréal a cédé sa place de sur des travaux, cités dans les références, menés avec mes collègues du LASER ${ }^{1}$. Cette synthèse se veut aussi une réflexion plus générale sur les défis de développement des régions québécoises. Le Québec est aujourd'hui une économie mature. Les défis de développement régional qui s'y présentent ne sont pas très différents de ceux des autres pays industrialisés, mais avec quelques éléments particuliers, le reflet surtout de l'étendue de son territoire et la présence d'industries hautement capitalisées en périphérie.

première métropole du Canada à Toronto, rupture qui marquait le début d'une longue période de déclin relatif. Cette rupture de tendance était la conséquence prévisible, et on pourrait dire «normale », de la refrancisation du Québec ${ }^{3}$; imposer le français et demeurer, en même temps, la capitale économique d'un pays aux trois quarts anglophone était impossible. La refrancisation de Montréal était nécessaire, mais il y avait un prix à payer. Une bonne partie de la vieille élite anglophone est partie avec ses sièges sociaux, ses capitaux et ses talents; ce qui n'a pas seulement eu pour effet d'affaiblir l'économie montréalaise, mais aussi de donner un coup de main inattendu à l'économie torontoise. Pendant trois décennies, la région de Toronto affichait une 
croissance nettement au-dessus de la moyenne nord-américaine.

Heureusement - pour Montréal, mais non pas pour Toronto - cette période est révolue. La perte de fonctions métropolitaines au profit de Toronto était un événement unique, le résultat d'une conjoncture politique particulière. En termes plus crus, les populations qui voulaient partir sont parties. Entre-temps, une nouvelle élite économique francophone est venue les remplacer. L'économie montréalaise s'est graduellement ajustée à son nouveau rôle de métropole d'une aire d'influence plus petite, mais maintenant bien à elle, protégée par la barrière de la langue et dynamisée par cette nouvelle élite. Sur plusieurs indicateurs économiques - sauf la taille (bien entendu) la métropole québécoise est en train aujourd'hui de rattraper la Ville-Reine. Cette transformation n'est pas uniquement le résultat d'une bonne performance de l'économie montréalaise, mais aussi de l'essoufflement de

\section{L'essor de l'Arc industriel québécois}

Dans les économies avancées, l'industrie fuit les grandes métropoles, devenues trop chères. La tertiarisation de Montréal a comme contrepartie la déconcentration de l'industrie manufacturière vers des villes moyennes, tendance qui touche surtout les industries de moyenne technologie, grandes consommatrices d'espace à la recherche d'une maind'œuvre de qualité (mais pas forcément super instruite), et sensibles par conséquent aux prix immobiliers et aux salaires. Depuis quelques années, l'emploi manufacturier croît plus rapidement à l'extérieur de la région métropolitaine de Montréal. Cependant, les entreprises manufacturières ne s'installent pas n'importe où. Elles chercheront, en règle générale, à ne pas trop s'éloigner des grandes métropoles et, en parallèle, à maximiser l'accès aux grands marchés, ce qui favorise le sud-est québécois. la croissance hors norme de l'économie torontoise, davantage frappée en plus par des chocs conjoncturels récents à cause de sa spécialisation dans le secteur financier et du sud ontarien dans l'industrie automobile.

Sauf pour la finance, où Toronto garde une avance inattaquable, Montréal se réaffirme aujourd'hui comme métropole dans plusieurs secteurs d'activité du tertiaire supérieur. Les grands bureaux de génie-conseil et les nombreuses entreprises de conception graphique par ordinateur en sont des exemples. Toutes proportions gardées, l'économie montréalaise repose aujourd'hui, autant que celle de Toronto, sur des fonctions métropolitaines.

Sauf pour la finance, où Toronto garde une avance inattaquable, Montréal se réaffirme aujourd'hui comme métropole dans plusieurs secteurs d'activité du tertiaire supérieur

Depuis quelques années, l'emploi manufacturier dans le Québec hors-Montréal croît plus vite que dans l'Ontario non-métropolitain (c'est-à-dire, à l'extérieur de la région de Toronto). Comment expliquer ce succès industriel du Québec non-métropolitain? Plusieurs éléments viennent à l'esprit, en commençant par les salaires plus bas au Québec, notamment dans des villes moyennes du sud-est québécois. Comparons Drummondville, à proximité de Montréal, à Guelph, à proximité de Toronto. Les gains médians (hommes ayant un travail à temps plein) étaient en 2006 presque $40 \%$ plus élevés à Guelph qu'à Drummondville ${ }^{4}$. À ceci s'joute la plus grande stabilité (la mobilité moindre) de la main-d'œuvre québécoise francophone, un avantage pour les entreprises pour lesquelles l'apprentissage sur le tas constitue un coût majeur. Bref, pour une entreprise à la 
recherche d'une main-d'œuvre stable avec une formation de base, mais pas forcément universitaire, Drummonville constitue le meilleur choix. De plus, à l'opposé du sud-est québécois, l'emprise dans le sud ontarien de l'industrie automobile, avec ses grandes usines et ses taux élevés de syndicalisation, ne favorise pas la création de PME locales. La proximité du Rustbelt américain n'aide pas non plus. Les villes de Détroit et de Buffalo ne sont guère des modèles de dynamisme. Le sud du Québec n'a pas à composer avec de telles voisines encombrantes. De plus, le sud ontarien ne possède aucun lien terrestre direct avec les États-Unis; le trafic doit emprunter un pont ou un bateau, ou encore passer par le Québec. Le sud-est du Québec a plus de chance: des dizaines de points de passage sillonnent sa frontière avec les États voisins.

Le moment est venu de présenter «l'Arc industriel du Québec ", une vaste bande de territoire qui s'étend grosso modo de St-Jeansur-Richelieu jusqu'aux portes de Rivière-duLoup, en passant par l'Estrie, les Bois-Francs, le Centre-du-Québec et la Beauce. Je ne suis pas le seul à évoquer le dynamisme industriel de cette partie du Québec. Proulx ${ }^{5}$ identifie ce qu'il appelle « le croissant manufacturier » qui épouse, en gros, les mêmes contours. Lemelin ${ }^{6}$ parle d'une zone encore plus vaste en forme de « $U$ » où les indicateurs de PIB (Produit intérieur brut) sont systématiquement audessus de la moyenne québécoise et dont les pôles urbains sont les régions de Montréal, de Sherbrooke et de Québec. Toutefois, l'Arc industriel québécois évoqué ici exclut expressément la région métropolitaine de Montréal.

Depuis une quinzaine années, c'est dans cet arc (ou croissant) que l'emploi manufacturier croît le plus rapidement. C'est aussi ici que l'on retrouve de nombreuses villes moyennes avec des fortes proportions d'emploi manufacturier. Il suffit de nommer des villes comme Granby, Victoriaville, Drummondville,
Cowansville, Ste-Marie et St-Georges-deBeauce, pour comprendre l'univers dont il est question. Plusieurs facteurs sont à l'origine de ce succès, dont des coûts compétitifs et l'accès au marché américain. Être localisé au sud plutôt qu'au nord du St-Laurent n'est pas sans importance. L'entreprise à Sherbrooke jouit d'un net avantage sur sa collègue de Chicoutimi où, en plus, les coûts de maind'œuvre sont plus élevés. Les salaires sont, en règle générale, plus élevés dans les régionsressources, résultat de la présence de grandes entreprises fortement capitalisées (alumineries, papeteries...), ce qui a pour effet de décourager l'émergence de PME exportatrices, phénomène que nous avons nommé le syndrome du rentier encombrant ${ }^{7}$. En 2006, les salaires étaient $20 \%$ plus élevés à Saguenay qu'à Sherbrooke (gains médians, hommes, temps plein), et $30 \%$ plus élevés qu'à Drummonville $^{8}$. Bref, pourquoi fonder une entreprise (exportatrice) à Saguenay alumineries, scieries et papeteries exceptées si vous pouvez le faire en Estrie ou en Beauce? En plus d'être loin des marchés, vous devrez payer des salaires plus élevés.

\section{Il est impossible de ne pas évoquer ce qu'il est convenu d'appeler le phénomène beauceron}

Si les villes du sud québécois ont, en règle générale, des marchés de travail plus compétitifs, c'est précisément à cause de l'absence de grandes entreprises « lourdes ». À cela s'ajoute l'héritage de l'industrie du textile et du vêtement qui a longtemps dominé l'économie des Cantons-de-l'Est et des régions avoisinantes. Comme en témoigne l'exemple de la Nouvelle-Angleterre voisine, les anciennes régions du textile ont, en règle générale, mieux réussi la reconversion vers l'économie moderne que les régions caractérisées par l'industrie lourde. Finalement, il est impossible de ne pas évoquer ce qu'il est convenu d'appeler le phénomène 
beauceron et l'émergence de l'entrepreneuriat québécois qui ne se limite pas, il faut le croire, à la Beauce. Son émergence dans cette partie du Québec n'est pas un hasard. En réunissant plusieurs traits - la proximité d'une grande ville (Montréal ou Québec), l'accès au marché américain, une main-d'œuvre loyale, des salaires et des coûts immobiliers compétitifs, un héritage industriel favorable - cette partie du Québec constitue un terroir particulièrement fertile pour des PME manufacturières.

L'essor de l'Arc industriel a comme pendant la métamorphose économique de la région de Québec. Depuis quelques années, la croissance de l'emploi y dépasse celle de la région montréalaise; cette croissance ne se fonde plus

\section{L'essor des économies résidentielles ${ }^{10}$}

Le vieillissement des populations est une autre tendance lourde des sociétés avancées. Le Québec ne possède ni Côte-d'Azur ni Sunbelt pour accueillir les jeunes retraités à la recherche du soleil. Le mouvement des populations retraitées et des professionnels mobiles ne provoquera pas au Québec un déplacement aussi marqué qu'en France, où le sud-ouest, traditionnellement périphérique, est aujourd'hui en plein essor. Pas de ruée non plus vers la Californie, qui a remplacé l'État de New-York comme l'État le plus populeux des États-Unis. Cependant, en matière de soleil, mer et espaces verts, les avantages sont relatifs. À défaut d'un «midi » à nous, les Québécois doivent se contenter de ce qu'ils ont, du moins pour ceux qui souhaitent (ou doivent) rester à l'intérieur des frontières du Québec.

Les principaux gagnants sont des territoires qui jouissent de certains avantages naturels (lacs, front maritime, montagne, climat moins rude...) et qui ne sont pas trop éloignés des grands centres. Ce deuxième critère joue non sur le secteur public. Québec est en train de changer de vocation, un peu à l'image de la région d'Ottawa qui, dans les années 1980, a vu naître sur son territoire un important foyer de haute technologie. Ribichesi et $a l^{9}$ constatent l'émergence de pôles technologiques dans la région, dont autour $\mathrm{du}$ complexe scientifique et dans le quartier StRoch, en pleine ébullition, qui commence à prendre des allures de l'axe de la rue StLaurent à Montréal, où logent des entreprises comme Ubisoft et Softimage. L'implantation à Québec d'activités de haute technologie est souvent le fait d'entreprises montréalaises en expansion à la recherche d'une main-d'œuvre stable et bien formée, mais avec des coûts salariaux et immobiliers plus faibles.

pas uniquement pour les personnes qui veulent garder un lien de travail avec la ville, mais aussi chez des retraités pour assurer l'accès à des services spécialisés (et aussi, parfois, à la parenté en ville). Autour de Montréal, cela continue à favoriser les Laurentides, Lanaudière, l'Outaouais proche et l'Estrie. Les résidents secondaires font partie de la base économique de villes comme Magog et Sutton. L'Estrie jouit, en ce sens, d'un avantage supplémentaire qui s'ajoute à son héritage industriel favorable et sa proximité à la frontière américaine.

Des zones d'économie résidentielle s'observent aussi autour d'Ottawa et de Québec. Autour de Québec, le mouvement est en train de gagner de nouveaux territoires, dont le littoral du Bas-St-Laurent. Il est possible que l'arrivée, quoique encore timide, des populations retraitées (ou préretraitées) dans le Bas-St-Laurent cache aussi un mouvement de retour $^{11}$. Une tendance similaire se devine en Gaspésie et dans les Îles. Par contre, elle ne semble pas être présente en Abitibi, sur la 
Côte-Nord et au Saguenay-Lac-St-Jean. À l'est de Tadoussac, grosso modo, l'attraction maritime joue, semble-t-il, uniquement au sud $\mathrm{du}$ St-Laurent. Pouvons-nous alors prévoir la «Laurentidisation» du Bas-du-Fleuve : un marché immobilier dominé par des résidences secondaires? Peut-être. Toutefois, elle se limite surtout au littoral, et n'est pas d'un grand secours pour l'arrière-pays (Témiscouata, Matapédia...) dont le déclin démographique semble irréversible.

\section{Le défi des vieux territoires industriels}

\section{Plusieurs MRC situées près du fleuve entre Québec et Montréal connaissent une crois- \\ Être situé proche de l'autoroute 20 ou 10 est infiniment plus utile que d'être situé sur le St-Laurent}

sance plutôt décevante

Une situation au bord du St-Laurent ne procure plus aucun avantage particulier, sauf pour les villes depuis quelques années, tendance qui se prolonge à l'ouest de Montréal pour certaines municipalités riveraines (dont Valleyfield). Comme nous l'avons proposé dans Apparicio et $a l^{12}$, la réponse se trouve sans doute dans le rôle historique du St.-Laurent comme corridor de transport des marchandises en vrac (minerai de fer, charbon, bauxite, troncs d'arbres...) qui, à son tour, a enfanté des industries lourdes. Le syndrome du rentier encombrant trouve un terrain fertile dans des collectivités dominées aujourd'hui ou par le passé par des grandes papeteries (on pense spontanément à Trois-Rivières), des alumineries et des sidérurgies (Sorel-Tracy). L'héritage légué par de telles industries ne se défait pas du jour au lendemain. Le Québec n'a pas l'équivalent de la Wallonie ou des Midlands anglais, qui continuent à connaître des difficultés à s'affranchir des mentalités d'une autre époque, mais le défi n'est pas moins réel pour plusieurs localités situées sur les rives du St Laurent, surtout sur la rive nord ${ }^{13}$.

\section{Le malheur des périphéries riches}

Il n'est pas utile ici de reprendre les analyses de Chenard et al ${ }^{11}$ ou de Polèse et Shearmur ${ }^{7}$, auxquelles je n'aurais pas grand-chose à ajouter, sauf pour rappeler que les périphéries québécoises doivent composer avec des défis dotées d'un port de conteneurs. Avant, cet axe fluvial était un atout. Aujourd'hui, les voies structurantes sont les autoroutes et, à un degré moindre, les lignes de chemin de fer. Être situé proche de l'autoroute 20 ou 10 est infiniment plus utile que d'être situé sur le St-Laurent. Le legs d'un passé industriel lourd - aux deux sens du terme - finira par s'estomper un jour, il faut l'espérer. Mais pour cela, il faut que la région possède d'autres atouts, dont notamment une localisation favorable. Il n'y a pas de raison pour que Trois-Rivières ne finisse pas un jour par retrouver une croissance comparable à celle de Montréal ou Québec. Mais cela passera nécessairement par un changement de mentalités.

Là où le bât blesse, c'est lorsque le syndrome $\mathrm{du}$ rentier encombrant s'ajoute à d'autres désavantages; ce qui nous conduit aux périphéries.

particuliers. Mes propos ici portent sur la différence entre des économies locales dominées par des grandes entreprises et celles où les PME sont davantage présentes, sous forme d'exploitants agricoles ou forestiers, de 
pêcheurs et d'autres entreprises familiales. Des grandes entreprises - des papeteries notamment - se retrouvent un peu partout, certes, mais les proportions ne sont pas partout les mêmes. Les défis auxquels font face les deux types d'économie locale ne sont pas les mêmes. J'aurais aussi pu parler de la distinction entre périphéries collectivistes et individualistes.

La dichotomie n'est pas parfaite, bien entendu. L'Abitibi se trouve entre les deux à cause de sa tradition minière, mais qui se traduit néanmoins par une culture entrepreneuriale différente de celle associée aux grandes alumineries de la Côte-Nord et du Saguenay ${ }^{14}$. L'Abitibi est un peu la périphérie Western du Québec, caractérisée par un certain esprit individualiste et rebelle (le berceau naguère du Crédit social), portrait renforcé par son rôle de région-relais entre la Baie-James et le sud. Il est peu probable que l'or et les diamants cessent un jour d'être en demande, nonobstant des fortes fluctuations. Il y aura toujours une base (irréductible) d'emplois dans la région, même si le plancher sera plus bas. Un raisonnement analogue s'applique à la Gaspésie et aux Îles, malgré les durs chocs du passé. Il y aura toujours une demande pour ses paysages et pour les produits de la mer. La force de travail demandée continuera à baisser, mais une rupture brutale est peu probable. La population s'est largement maintenue aux Îlesde-la-Madeleine; si déclin il y a, il se fera graduellement.

La situation de la Côte-Nord et du Saguenay ${ }^{15}$ est toute autre; l'emploi y repose davantage sur des activités de transformation alumineries surtout - dont les avantages se fondent sur des technologies en vigueur et sur des choix politiques. L'emploi y est davantage concentré dans quelques grandes usines. De telles économies sont davantage sujettes à des chocs brutaux. La survie à long terme des alumineries repose sur des paris techno- logiques : que l'aluminium ne sera pas un jour remplacé par d'autres matériaux comme ce fut naguère le cas pour l'acier; que les procédés de production restent énergivores; que les coûts de transport de l'électricité restent élevés. Aucune de ces données ne semble menacée pour le moment; quoique la chute brutale du prix d'aluminium lors de la récession amorcée en 2008 démontre à nouveau les dangers potentiels d'une trop forte spécialisation dans un seul secteur industriel (un peu à l'image du sud-ontarien actuel). Mais la rentabilité des alumineries repose aussi sur un choix politique; à savoir, le choix de l'État québécois, par l'intermédiaire d'ententes entre Hydro-Québec et les alumineries, de leur vendre l'électricité à des prix en dessous du marché. Cette subvention implicite, dénoncée par certains économistes ${ }^{16}$, fait une base économique davantage sensible à des changements d'humeur politique.

Cette partie du Québec connaît beaucoup de difficulté à effectuer le passage vers une économie fondée sur des PME diversifiées, contrepartie $\mathrm{du}$ syndrome $\mathrm{du}$ rentier encombrant. Le défi est de taille : comment, avec des marchés de travail artificiellement chers, faire émerger des entreprises dans d'autres secteurs d'activité? Le danger : il n'y aura pas de solution de rechange le jour où l'usine fermera. L'usine crée la richesse aujourd'hui, mais hypothèque l'avenir. Voici le dilemme. La différence avec le Bas-StLaurent est révélatrice. Il réussit mieux depuis quelques années que d'autres régions périphériques à s'affranchir de l'emprise des industries de première transformation. L'absence de grandes alumineries y est sans doute pour quelque chose. Ajouté aux avantages résidentiels que lui procure son littoral, il faudra peut-être cesser de classer le Bas-St-Laurent dans la famille des régions périphériques (ou régions ressources), ce qui est finalement une bonne nouvelle. 


\section{Conclusion}

Le tableau présenté ici est sensiblement différent de celui d'un Québec « des régions », éternel perdant, face à une Montréal trop puissante. La réalité est moins tranchée et disons-le - plus encourageante. Une bonne partie du Québec méridional, nommé l'Arc industriel québécois, connaît depuis quelque temps une meilleure performance que la métropole, à laquelle s'ajoute le nouveau dynamisme de la région de Québec. Des villes moyennes comme Drummondville, Victoriaville, Granby et St-Georges-de-Beauce, pour ne nommer que celles-là, font preuve d'un dynamisme industriel surprenant, qui repose sur une combinaison de facteurs : des salaires compétitifs, un héritage industriel positif, la proximité de Montréal (ou de Québec), l'accès au marché américain, auxquels s'ajoute aussi une petite dose d'esprit d'entrepreneurial proprement québécois.

Cette croissance industrielle «en région » ne se fait pas contre Montréal. Elle en est un complément naturel. La force de Montréal dans le tertiaire supérieur et la force industrielle, en parallèle, des villes moyennes sont deux côtés de la même médaille. L'essor du tertiaire supérieur dans la métropole y fait monter les coûts (salaires, immobilier, congestion...), ce qui la rend moins intéressante pour beaucoup d'activités manufacturières; mais, d'autre part, la proximité de Montréal - avec ses nombreux services - constitue un avantage pour les villes moyennes du sud-est québécois, qui sont les clients naturels des services supérieurs de Montréal. Les préférences résidentielles des populations retraitées et préretraitées ainsi que de certaines classes professionnelles favorisent également le Québec méridional, dont notamment les Laurentides et l'Estrie, mais aussi le littoral du St-Laurent à l'est de Québec.

Cette croissance industrielle « en région » ne se fait pas contre Montréal

Le dynamisme industriel (mais aussi récréatif) $\mathrm{du}$ sud québécois nous fait voir la problématique régionale québécoise d'un autre œil. Ce n'est pas dans l'opposition MontréalRégions que se joue la concurrence entre régions québécoises. Les régions périphériques ne sont pas en concurrence avec la région montréalaise. Pour la plupart des PME industrielles, le choix n'est pas entre, disons, Chicoutimi ou Rouyn-Noranda et Montréal, mais entre Chicoutimi ou Rouyn-Noranda et des villes moyennes du sud-est québécois. Pour fonder un atelier de fabrication de meubles, à titre d'exemple, les vraies concurrentes ne sont pas les municipalités de la région métropolitaine de Montréal, mais bien Granby, Ste-Marie-de-Beauce et leurs sœurs de l'Arc industriel québécois.

Le contraste entre les villes moyennes du sudest québécois et les collectivités périphériques, dominées par des grandes entreprises, est de plus en plus marqué. D'un côté, des avantages de localisation et des marchés de travail compétitifs qui favorisent l'éclosion de l'entrepreneuriat local et, de l'autre côté, un entrepreneuriat local bloqué, non pas seulement par la distance, mais aussi par des marchés de travail dysfonctionnels. Les défis de développement local ne sont manifestement pas les mêmes des deux côtés. 


\section{Notes et références}

* Titulaire de la Chaire de recherche senior du Canada en Études urbaines et régionales. L'auteur remercie le Fonds québécois de la recherche sur la science et la culture (FQRSC) et le Conseil de recherche en sciences humaines du Canada (CRSHC) de leur aide financière

1 Polèse, M. (2009), «Les nouvelles dynamiques régionales de l'économie québécoise : cinq tendances », Recherches sociographiques, Vol. L, no. 1 : 9-39

2 Laboratoire d'analyse spatiale et d'économie régionale, logé à l'INRS Urbanisation, Culture et Société à Montréal. La majorité des études citées sont disponibles sur le site du LASER : http://laser.ucs.inrs.ca/

3 Polèse, M. (1990), «La thèse du déclin économique de Montréal, revue et corrigée », L'Actualité économique, vol. 66 , no 2 : 133-146

4 StatCan (2008), Revue chronologique de la population active - 2007, Statistique Canada (format CD) Cat. 71F0004XCB

5 Proulx, M.-U. (2006), «La mouvance contemporaine des territoires: la logique spatiale de l'économie du Québec », Recherches sociographiques, Vol. XLVII, No.3 : 475-502; numéro spécial sur le développement territorial sous la direction de Bruno Jean

6 Lemelin, A. (2000), Montréal dans l'économie du Québec, INRS Urbanisation, Culture et Société : http://www.ucs.inrs.ca/pdf/Montreal.pdf

7 Polèse, M. et R. Shearmur (2002), La périphérie face à l'économie du savoir: la dynamique spatiale de l'économie canadienne et l'avenir des régions non métropolitaines du Québec et du Canada atlantique, INRSUCS et ICRDR. Montréal et Moncton : http://www.ucs.inrs.ca/default.asp?p=res

8 StatCan (2008a), Profils des communautés de 2006, Statistique Canada, Cat. 92-591-XWF Internet: http://www.statcan.ca/bsolc/francais/bsolc?catno=92-591-XWF

9 Ribichesi, C., M. Polèse et R. Shearmur (2008), L'économie du savoir dans la ville de Québec : revue de la littérature et analyses empirique, INRS Urbanisation, Culture et Société, Montréal

10 J'emprunte cette expression à Davezies (2008) qui l'emploie pour designer des territoires en France dont l'économie repose désormais sur des choix de résidence (de jeunes retraités et de professionnels ) plutôt que sur des activités productives d'exportation.

11 Chenard, Ph., M. Polèse et R. Shearmur (2005), L'évolution économique et démographique et perspectives de développement des régions périphériques du Québec, INRS Urbanisation, Culture et Société: http://www.ucs.inrs.ca/pdf/SECOR.pdf

12 Apparicio, Ph., M. Polèse et R. Shearmur (2008), «Une histoire de résidus : à propos de la stabilité et la volatilité des facteurs de croissance régionale, Canada 1971-2001 », Inédits 2008-8, INRS Urbanisation, Culture et Société http://www.ucs.inrs.ca/default.asp?p=pl

Apparicio, Ph., G. Dussault, M. Polèse et R. Shearmur (2007), Infrastructures de transports et développement économique local. Étude de la relation entre accessibilité continentale et croissance locale de l'emploi, Canada, 1971-2001, INRS Urbanisation, Culture et Société : http://projetic.ucs.inrs.ca/

13 Le lien avec le côté « nord » n'est pas difficile à comprendre. Les ressources, dont le bois, y étaient plus proches et plus accessibles. L'électricité coûte cher à transporter; passer des lignes de transmission sur le fleuve est un coût à éviter.

14 À Rouyn-Noranda, la fonderie de la Noranda, qui autrefois dominait totalement le paysage local, n'est plus l'employeur principal. De plus, il est presque inévitable qu'elle finira par fermer ses portes dans les années à venir.

15 Je fais consciemment abstraction du Lac-St-Jean (à l'ouest d'Alma), car sa base économique repose davantage sur l'exploitation de la terre (agriculture) et de la forêt. 
Bernard, J.-T. et M. Bélanger (2007), Aluminium : des subventions annuelles de 336000 \$ par emploi pour 30 ans, Département d'économique, Université Laval, Québec : http://www.asdeq.org/activites/comite-politiquespubliques/pdf/2007/Alumineries-Subventions-336000-par-emploi.pdf

Davezies, L. (2008), La république et ses territoires : La circulation invisible des richesses, Seuil, Paris

Henderson, V., Z. Shalizi, et A.J. Venables (2001), «Geography and development », Journal of Economic Geography, $1: 81-205$

Polèse, M. et R. Shearmur (2003), « Pourquoi Toronto a surclassé Montréal au sommet de la hiérarchie urbaine canadienne? L'impact des différences culturelles sur la dynamique spatiale des services supérieurs », Géographie Économie Société, 5 (3-4) : 399-420

Shearmur, R. et M. Polèse (2005), La géographie du niveau de vie au Canada, 1971-2001, INRS Urbanisation, Culture et Société : http://www.ucs.inrs.ca/pdf/GeographieNiveauVie.pdf 


\section{Publicité Prog. cycles supérieurs}

